\title{
Enhancement of the near-side component in quasiadiabatic calculations of the ${ }^{66} \mathrm{Zn}(d, p){ }^{67} \mathrm{Zn}$ reaction
}

\author{
E. J. Stephenson, A. D. Bacher, G. P. A. Berg, V. R. Cupps, C. C. Foster, N. Hodiwalla, \\ P. Li, J. Lisantti, D. A. Low, D. W. Miller, C. Olmer, A. K. Opper, B. K. Park, \\ R. Sawafta, and S. W. Wissink \\ Indiana University Cyclotron Facility, Bloomington, Indiana 47405 \\ J. A. Tostevin, D. A. Coley, ${ }^{*}$ and R. C. Johnson \\ Department of Physics, University of Surrey, Guildford, Surrey GU2 5XH, United Kingdom
}

(Received 9 April 1990)

\begin{abstract}
Measurements of the spin dependence of large $l, j=l-\frac{1}{2}$, transfer reactions at medium energies provide a stringent test of three-body reaction models. We report measurements of the cross section $(d \sigma / d \Omega)$, vector $\left(A_{y}\right)$ and tensor $\left(A_{y y}\right)$ analyzing powers, and outgoing proton polarization $(p)$ angular distributions $\left(\theta_{\text {c. m. }}=6.9^{\circ}-82.3^{\circ}\right)$ for the $l=3, j^{\pi}=\frac{5}{2}^{-}$ground-state transition in ${ }^{66} \mathrm{Zn}(d, p)^{67} \mathrm{Zn}$ measured with $88.2-\mathrm{MeV}$ deuterons. Adiabatic model calculations fail to describe either interference oscillations in the data or departures from the simple theoretical relationships, $1+3 p+2 A_{y y}=0$ and $2+A_{y y}+3 A_{y}=0$, that are expected to hold for a far-side-dominated reaction. The quasiadiabatic model, in which the center-of-mass energy in breakup configurations is not constrained to be degenerate with the elastic channel, is able to reproduce qualitatively these features of the data.
\end{abstract}

\section{INTRODUCTION}

The coupled discretized continuum channels (CDCC) technique for the treatment of breakup effects in the deuteron-nucleus system has recently been the subject of two extensive review articles. ${ }^{1,2}$ While the CDCC method is able to produce accurate three-body wave functions, including the effects of the nucleon-target spin-orbit interactions, these wave functions have yet to be incorporated fully into transfer reaction calculations. Only zero-range transfer reaction calculations have been carried out so far, and these neglect the entrance channel spin dependence. At the energies of interest here, it is vital to include this spin dependence in order to reproduce even the qualitative features of the reaction data.

As a result, calculations of $(d, p)$ transfer reactions often employ the adiabatic distorted-wave approximation (ADWA). ${ }^{3}$ If, however, we are to use such calculations routinely to extract spectroscopic strengths or other features of the reaction, we must be confident that they correctly describe the major aspects of the reaction mechanism. A thorough study of the ${ }^{116} \operatorname{Sn}(d, p){ }^{117} \operatorname{Sn}\left(E_{d}=79\right.$ $\mathrm{MeV}$ ) reaction was undertaken to investigate the accuracy of the ADWA for the momentum mismatched $l=0$ ground-state transition, ${ }^{3}$ which was expected to be poorly described at intermediate energies. Instead, the adiabatic model had much more difficulty with the better-matched $j^{\pi}=\frac{7}{2}^{+}, l=4$ transition..$^{4,5}$ The inclusion in these calculations of finite-range effects, including transfer from the deuteron $D$ state, and nonlocality corrections, while making significant improvements in the reaction observables, did not result in good agreement with the data, especially with the angular distributions of the analyzing powers.
Further investigation showed that the transfer reaction amplitudes obtained using the ADWA model described flux that flowed mainly around the far side of the nucleus (the side away from the detector), a selectivity that arose from the attractiveness of the nuclear optical potential. In addition, this flux and the transferred neutron orbits populated were coplanar with the asymptotic deuteron and proton momenta. ${ }^{4}$ This feature proved to be an excellent guide to the observed $j$-transfer dependence of the reaction vector $\left(A_{y}\right)$ and tensor $\left(A_{y y}\right)$ analyzing powers. ${ }^{4}$ For $j=l-\frac{1}{2}$ transitions, the dominance of flux on the far side allows only two elements of the transition matrix (as a function of the spin-projection quantum numbers) to contribute significantly, with the result that simple relationships exist among the spin observables. ${ }^{5}$ In particular,

$$
\begin{aligned}
& 1+3 p+2 A_{y y}=0, \\
& 2+A_{y y}+3 A_{y}=0,
\end{aligned}
$$

where $p$ is the outgoing proton polarization, become exact in the limit that only those two amplitudes are nonvanishing. The latter relation is broken whenever there is a spin flip in the entrance (deuteron) channel. Transfer from the deuteron $D$ state, a process included in the ADWA model, is one such spin-flip mechanism. However, for the $\frac{7}{2}^{+}$transition in ${ }^{116} \mathrm{Sn}(d, p)^{117} \mathrm{Sn}$, the violation of Eq. (2) obtained empirically substantially exceeded the contribution calculated due to the $D$ state, ${ }^{5}$ but it was not clear to what extent each of the assumptions was violated. Equation (1), on the other hand, holds independently of such spin-flip considerations, requiring only far-side dominance and parity conservation for its validity. ${ }^{5}$ It 
was thus deemed important to investigate another reaction for which measurements could be performed to check Eq. (1).

The ${ }^{66} \mathrm{Zn}(d, p){ }^{67} \mathrm{Zn}$ reaction was chosen because the ground-state $\left(j^{\pi}=\frac{5}{2}^{-}\right)$transition is of $j=l-\frac{1}{2}$ character, and the transition connects two stable isotopes. This means that measurements of the outgoing proton polarization could be obtained from the equivalent analyzing power in the time-reversed ${ }^{67} \mathrm{Zn}(p, d)^{66} \mathrm{Zn}$ reaction at an appropriate energy. The spectroscopic factor for the transition is also large enough (between 0.2 and 0.3 ) that we did not anticipate any complications from coupling to other discrete transitions. This new set of measurements would allow us to check whether other spin-flip processes not presently included in the calculations, such as tensor distortions in the deuteron channel and transfer out of $D$ or higher $l$ states in the continuum, were responsible for the disagreement. The deuteron energy of $88.2 \mathrm{MeV}$ was selected as a balance between the desire to emphasize the far-side character of the transition by raising the bombarding energy and the capabilities of the Indiana University Cyclotron Facility (IUCF) cyclotron for stable operation.

A second clue to the nature of the reaction mechanism difficulty came from the observation that interference patterns with a period compatible with the diameter of the nucleus were present in the measured analyzing power angular distributions and nearly absent in the calculations. This called into question the theoretical property of far-side dominance (but not necessarily coplanarity), suggesting the presence of a near-side amplitude comparable in strength to the one from the nuclear far side. If there are only two large far-side amplitudes, then it should be possible to estimate the size of the missing near-side piece directly by fitting an empirical near-side amplitude to the interference pattern present in

$$
(d \sigma / d \Omega)_{0}=(d \sigma / d \Omega)\left(1-A_{y y}\right) / 3
$$

and

$$
(d \sigma / d \Omega)_{-1}=(d \sigma / d \Omega)\left(1-3 A_{y} / 2+A_{y y} / 2\right) / 3,
$$

the two parts of the cross section (the index refers to the entrance channel projection of the deuteron spin along an axis perpendicular to the reaction plane) that originate from the two amplitudes. ${ }^{6}$ A model was constructed that used analytic forms for the near-side and far-side terms consistent with a single-pole approximation to the reaction radial integrals (in partial-wave space). From the spin coupling of the near- and far-side terms and the fitting parameters, it was concluded that the missing near-side amplitude arose from nearly central deuteronnucleus collisions and exhibited a very large dependence on the spin-projection quantum number. To see whether this characterization was peculiar to the ${ }^{116} \mathrm{Sn}$ transition, a large range of scattering angles was included in the ${ }^{66} \mathrm{Zn}$ study so that a similar empirical estimate could be made of the missing near-side amplitude.

The empirical near-side amplitude has quantum numbers which, in a semiclassical picture, suggest that large relative neutron-proton momenta are involved at the point of transfer, since, after transfer, the neutron and proton orbit the nucleus in opposite directions. This puts in jeopardy the very basis of the adiabatic approximation, namely the assumed degeneracy of the $n-p$ center-of-mass energy in the breakup configurations with that of the incident bound deuteron. Removing this assumption would allow the $n-p$ center of mass to move, on average, with an energy less than that of the bound deuteron in the calculation of the breakup components in the threebody wave function. A technique for lifting this assumption has been described by Amakawa, Austern, and Vincent, ${ }^{7}$ which they name the quasiadiabatic model. We have extended their technique to include the effects of nucleon spin-orbit interactions and to allow the $n-p$ centerof-mass energy to depend on the center-of-mass radius and partial-wave number. In this paper we present the first calculations of this sort in zero-range approximation. Despite this simplification, we will show that the changes to the $n-p$ energetics are significant, and introduce nearside amplitudes of a size comparable to those required by the measurements. Thus both the interference pattern in the analyzing power and the deviations from Eqs. (1) and (2) appear to be understood within the context of a three-body model of the reaction dynamics.

To estimate the outgoing proton distorted waves, we also measured angular distributions of cross section and analyzing power for proton scattering from ${ }^{67} \mathrm{Zn}$ at 91.8 $\mathrm{MeV}$. The experimental details of this and the transfer reaction measurements are given in Sec. II. The formal aspects of the three-body models used and a derivation of the quasiadiabatic formulas are presented in Sec. III. The extraction of a suitable set of final-state optical potential parameters for the proton elastic-scattering measurements is described in Sec. IV. To set the basis for comparison, standard ADWA reaction calculations are presented in Sec. V. The interference patterns present in $(d \sigma / d \Omega)_{0}$ and $(d \sigma / d \Omega)_{-1}$ are analyzed in Sec. VI to yield an estimate of the near-side amplitude required empirically. This estimate is compared with the near-side components present in both the ADWA and quasiadiabatic models in Sec. VII. Also in Sec. VII, the new quasiadiabatic calculations are compared with the cross section and analyzing power angular distributions, as well as the deviations from Eqs. (1) and (2).

\section{EXPERIMENTAL CONSIDERATIONS}

The polarized proton and deuteron beams needed for this experiment were generated by an atomic beam source containing both weak- and strong-field transition units, as reviewed in Ref. 8 . The output from the ion source was typically $6 \mu \mathrm{A}$.

Beam polarization measurements were made in the transfer line between the two IUCF separated-sector cyclotrons, where the beam typically had $8 \%$ of its final energy. For protons, elastic scattering from ${ }^{4} \mathrm{He}$ at $\theta_{\text {lab }}=112^{\circ}$ was used as the analyzer. The analyzing power is known from a phase-shift analysis of measurements for the $p+{ }^{4} \mathrm{He}$ system. ${ }^{9}$ The resulting polarization measurements were consistent over time with their average value, which was $0.76 \pm 0.01$ for spin up and $-0.74 \pm 0.02$ for 
spin down. To cancel the effects of slow changes in experimental conditions (beam energy, beam spot position, detector threshold, etc.), the spin state was reversed periodically with an interval of 10-30 s in each spin state.

For deuterons, the analyzing reaction was ${ }^{3} \mathrm{He}(d, p){ }^{4} \mathrm{He}$, observed with similar geometrical constraints at a scattering angle of $\theta_{\mathrm{lab}}=30^{\circ}$. The calibration of the vector and tensor analyzing powers of the polarimeter, and the extraction of the beam polarization, are described in Ref. 10. Four polarized spin states were used [where maximally $\left(p_{y}, p_{y y}\right)=\left(\frac{2}{3}, 0\right),\left(-\frac{2}{3}, 0\right),\left(-\frac{1}{3}, 1\right)$, and $\left.\left(-\frac{1}{3},-1\right)\right]$. The beam polarizations were again constant with time and typically $81 \%$ of the maximum values.

The cyclotrons were tuned for $88.2-\mathrm{MeV}$ deuteron energy and $91.8-\mathrm{MeV}$ proton energy, values chosen to produce comparable center-of-mass energies for the $(d, p)$ and ( $p, d)$ reactions when the ground-state $Q$ value is taken into account.

The ${ }^{66} \mathrm{Zn}$ and ${ }^{67} \mathrm{Zn}$ targets were self-supporting foils made of enriched $(98.8 \%$ and $93.1 \%$, respectively) material and rolled to thicknesses of $6.75 \mathrm{mg} / \mathrm{cm}^{2}\left({ }^{66} \mathrm{Zn}\right)$ and 7.35 or $15.53 \mathrm{mg} / \mathrm{cm}^{2}\left({ }^{67} \mathrm{Zn}\right)$. The target thicknesses were measured to a precision of 5-7\% by observing the energy loss of $\alpha$ particles from the ${ }^{232} \mathrm{Th}$ decay chain passing through the area of the target sampled by the cyclotron beam spot.

The reaction products were detected with the IUCF K600 magnetic spectrometer system. Dispersion matching techniques were used to achieve a typical resolution of $40 \mathrm{keV}$ at the focal plane detector. At large scattering angles, the tilt of the target to the beam was chosen so that differences in the deuteron and proton energy losses in traversing the target were also canceled. ${ }^{11}$

The focal plane detector system consisted of two vertical drift wire chambers ${ }^{12}$ with $6-\mathrm{mm}$ sense-wire spacing and a wire plane separation of $10.6 \mathrm{~mm}$. Start timing for the wire chambers and pulse-height information for particle identification were provided by two plastic scintillation detectors mounted behind the second wire chamber. A focal plane position spectrum for the ${ }^{66} \mathrm{Zn}(d, p){ }^{67} \mathrm{Zn}$ reaction is shown in Fig. 1, where the ground-state peak is clearly separated from the first and second excited states of ${ }^{67} \mathrm{Zn}$. In some cases it was necessary to subtract the tail of the first excited state from the region of the ground state before a peak area was calculated. This subtraction was made using an empirical peak shape modeled on the strong $\frac{9}{2}^{+}$excited state at $604 \mathrm{keV}$. Peak areas were corrected for dead time in the electronics and computer interface, and for events lost due to processing errors originating from difficulties with the wire chamber information. The sum of all such corrections was less than $15 \%$ of the peak area.

Cross sections and analyzing powers were calculated from peak areas and integrated beam currents obtained with each polarization state. At the larger scattering angles, the unpolarized and $\left(p_{y}, p_{y y}\right)=\left(-\frac{2}{3}, 0\right)$ states were eliminated from the experimental protocol to save time.

Errors shown in subsequent graphs are statistical, and represent a reasonable estimate of the point-to-point random variations within a particular angular distribution. In addition, the cross section may be in error by a scale

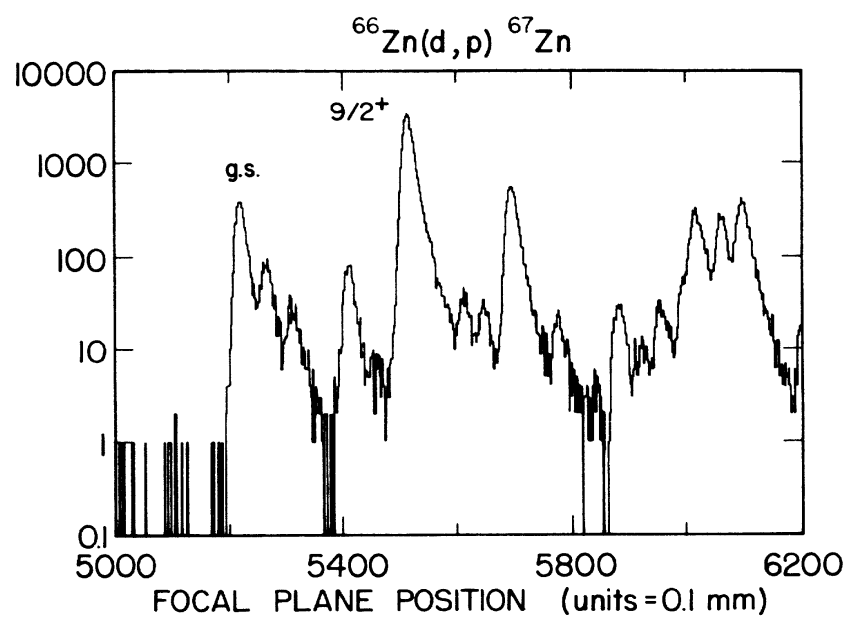

FIG. 1. A position spectrum from the focal plane detector of the K600 magnetic spectrometer. The horizontal axis gives position in units of $0.01 \mathrm{~mm}$, and the vertical axis gives counts per channel. The ground state and $\frac{9}{2}^{+}$states are indicated.

factor of $10 \%$ due to target thickness uncertainties, errors from the beam integration, and difficulties with estimating the electronic and wire chamber processing efficiencies. This error is consistent with a comparison of the $(d, p)$ and $(p, d)$ cross sections through detailed balance, which suggests that the $(d, p)$ cross section is larger than expected from its $(p, d)$ counterpart by a factor of $1.09 \pm 0.08$. Scale factor errors in the polarimeter analyzing powers are $\pm 1 \%$ for protons and $\pm 3 \%$ for deuterons. The polarizations on target may be uncertain by as much as $5 \%$ due to precession of the quantization axis through a small angle away from the vertical direction by longitudinal magnetic fields in the cyclotron.

\section{THE THREE-BODY MODELS}

We discuss the deuteron-nucleus system within the three-body $(n+p+$ target $)$ model. The transition amplitude for the $(d, p)$ reaction is thus denoted

$$
T\left(\sigma_{p} m \leftarrow \sigma_{d}\right)=\left\langle\chi_{\sigma_{p}}^{(-)} \phi_{j l}^{m}\left|V_{n p}\right| \psi_{\sigma_{d}}\right\rangle,
$$

where $\chi_{\sigma_{p}}^{(-)}$is the outgoing proton distorted wave, $\phi_{j l}^{m}$ the transferred neutron bound-state wave function, and $\psi_{\sigma_{d}}$ the exact three-body wave function for the deuteronnucleus system. Thus $\psi_{\sigma_{d}}$ satisfies the Schrödinger equation

$$
\left[E-H_{n p}-T_{R}-U(\mathbf{r}, \mathbf{R})\right] \psi_{\sigma_{d}}(\mathbf{r}, \mathbf{R})=0,
$$

where $H_{n p}$ is the $n-p$ relative motion Hamiltonian, $T_{R}$ is the $n-p$ center-of-mass kinetic-energy operator, and $U(\mathbf{r}, \mathbf{R})$ is the sum

$$
U(\mathbf{r}, \mathbf{R})=U_{n}(\mathbf{r}, \mathbf{R})+U_{p}(\mathbf{r}, \mathbf{R})
$$

of the neutron- and proton-target optical potentials evaluated at half the incident deuteron energy. The nucleon optical potentials will be denoted 


$$
U_{i}(\mathbf{r}, \mathbf{R})=U_{i}^{c}(|\mathbf{R} \pm \mathbf{r} / 2|)+U_{i}^{\mathrm{so}}(|\mathbf{R} \pm \mathbf{r} / 2|) l_{i} \cdot \sigma_{i}
$$

where the plus and minus signs relate to the proton and neutron, respectively. The purpose of three-body models of the transfer reaction, be they adiabatic, quasiadiabatic, or CDCC methods, is to provide an approximate solution for the wave function $\psi_{\sigma_{d}}$ for the evaluation of the transition amplitude of Eq. (3). While the CDCC technique in principle provides the most detailed and complete calculation of the three-body wave function, including the effects of breakup to $n-p$ relative $D$ waves, available calculations do not include this effect or the spin-orbit distortions within a transfer reaction calculation. ${ }^{13}$ We will concentrate here on corrections to the adiabatic approximation, including fully the effects of the spin-orbit interaction in the case of $S$-wave breakup. We note that in the adiabatic approximation $D$-wave breakup makes no contribution to the transfer amplitude in the zero-range limit.

\section{A. The adiabatic approximation}

A considerable simplification in the solution of the three-body equation is achieved using the adiabatic approximation. ${ }^{14}$ This makes the replacement $H_{n p} \rightarrow-\epsilon_{d}$, the deuteron binding energy, in Eq. (4), under the assumption that the dominant breakup configurations are states of low relative $n-p$ energy. Thus Eq. (4) reads

$$
\left[E_{\mathrm{c} . \mathrm{m} .}-T_{R}-U(\mathbf{r}, \mathbf{R})\right] \psi_{\sigma_{d}}^{A d}(\mathbf{r}, \mathbf{R})=0,
$$

where $E_{\text {c.m. }}=E+\epsilon_{d}$ is the energy of the incident deuteron in the center-of-mass frame, and

$$
\psi_{\sigma_{d}}^{A d}(\mathbf{r}, \mathbf{R})=\sum_{\sigma_{d}^{\prime}} \psi_{\sigma_{d}^{\prime} \sigma_{d}}^{A d}(\mathbf{r}, \mathbf{R}) \phi_{d}^{\sigma_{d}^{\prime}}(\mathbf{r})
$$

All $n-p$ relative motion configurations in $\psi_{\sigma_{d}}^{A d}$ are thus assumed to have the same center-of-mass energy. For the evaluation of a transfer reaction amplitude, $\psi_{\sigma_{d}}^{A d}$ is required only for those values of $r$ for which the $n-p$ interaction $V_{n p}$ is significant. Johnson and Tandy ${ }^{15}$ showed that the adiabatic approximation for $(d, p)$ reactions could also be regarded as the first term in an expansion of the three-body wave function $\psi_{\sigma_{d}}(\mathbf{r}, \mathbf{R})$ in terms of Sturmian states. This leads, for values of $r$ inside the range of $V_{n p}$, to

$$
\psi_{\sigma_{d}}^{A d}(\mathbf{r}, \mathbf{R}) \approx \bar{\psi}_{\sigma_{d}}^{A d}(\mathbf{r}, \mathbf{R})=\sum_{\sigma_{d}^{\prime}} \bar{\psi}_{\sigma_{d}^{\prime} \sigma_{d}}^{A d}(\mathbf{R}) \phi_{d}^{\sigma_{d}^{\prime}(\mathbf{r})},
$$

where $\bar{\psi}_{\sigma_{d}}^{A d}$ is calculated from the distorted-wave-like equation

$$
\left[E_{\text {c. m. }}-T_{R}-U^{A d}(\mathbf{R})\right] \bar{\psi}_{\sigma_{d}}^{A d}(\mathbf{r}, \mathbf{R})=0
$$

and the distorting interaction $U^{A d}$ is

$$
U^{A d}(\mathbf{R})=\left\langle\phi_{d}\left|V_{n p} U(\mathbf{r}, \mathbf{R})\right| \phi_{d}\right\rangle /\left\langle\phi_{d}\left|V_{n p}\right| \phi_{d}\right\rangle .
$$

Equation (9) is particularly attractive in that, numerically, the transfer reaction calculations take on the traditional distorted-wave Born approximation form. It fol- lows that finite-range effects, including the deuteron ground-state $D$-state component, can be readily included. Such calculations are referred to as ADWA calculations in this paper.

An alternative approach to the adiabatic approximation is to solve Eq. (7) directly. This is straightforward, since the dynamical dependence of the equation upon $r$, in $H_{n p}$, has been removed. We will make use of this method in our treatment of the quasiadiabatic approximation in the following subsection.

\section{B. The quasiadiabatic approximation}

The quasiadiabatic approximation of Amakawa, Austern, and Vincent ${ }^{7}$ provides the vehicle with which to improve our description of the center-of-mass motion of the $n-p$ pair. Specifically, it allows us to break the assumed degeneracy of the $n-p$ center-of-mass energy of all breakup configurations inherent in the adiabatic approximation, Eq. (7), above. We provide a brief derivation of the quasiadiabatic equations used since, unlike Amakawa, Austern, and Vincent, we will include the effects of nucleon spin-orbit forces consistently under the restriction to $S$-wave relative $n-p$ configurations.

With only $S$-wave $n-p$ relative motion, the angular average of $\mathrm{Eq}$. (4) can be taken to give

$$
\left[E-H_{n p}-T_{R}-\widetilde{U}(r, \mathbf{R})\right] \psi_{\sigma_{d}}(r, \mathbf{R})=0,
$$

where

$$
\widetilde{U}(r, \mathbf{R})=\frac{1}{4 \pi} \int d \Omega_{r} U(\mathbf{r}, \mathbf{R}) .
$$

This interaction consists of central and spin-orbit contributions

$$
\widetilde{U}(r, \mathbf{R})=\widetilde{U}^{c}(r, R)+\widetilde{U}^{\text {so }}(r, R) \mathbf{L} \cdot \mathbf{S},
$$

where $\mathbf{L}=-i \mathbf{R} \times \nabla_{R}$ and $\mathbf{S}=\frac{1}{2}\left(\boldsymbol{\sigma}_{p}+\boldsymbol{\sigma}_{n}\right)$ are the $n-p$ center-of-mass orbital angular momentum and $n-p$ total spin operators, respectively. Explicitly we have

$$
\begin{aligned}
& \widetilde{U}^{c}(r, R)=\frac{1}{2} \int_{-1}^{1} d \mu\left[U_{p}^{c}(x)+U_{n}^{c}(x)\right], \\
& \widetilde{U}^{\mathrm{so}}(r, R)=\frac{1}{2} \int_{-1}^{1} d \mu U_{p}^{\mathrm{so}}(x)\left(1+\frac{r \mu}{2 R}\right),
\end{aligned}
$$

where

$$
x=\left(R^{2}+r^{2} / 4+r R \mu\right)^{1 / 2},
$$

and $\mu$ is the cosine of the angle between $r$ and $R$. To proceed, we decompose the solution $\psi_{\sigma_{d}}$ of Eq. (12) into its elastic and inelastic (breakup) components by projection, i.e.,

$$
\psi_{\sigma_{d}}^{\mathrm{el}}(r, \mathbf{R})=\sum_{\sigma_{d}^{\prime}} \psi_{\sigma_{d}^{\prime} \sigma_{d}}^{\mathrm{el}}(\mathbf{R}) \phi_{d}^{\sigma_{d}^{\prime}}(r)
$$

where

$$
\psi_{\sigma_{d}^{\prime} \sigma_{d}}^{\mathrm{el}}(\mathbf{R})=\int_{0}^{\infty} d r r^{2} \phi_{d}^{\sigma_{d}^{\prime}}(r) \psi_{\sigma_{d}}(r, \mathbf{R})
$$

and we have normalized the ( $S$-state) deuteron wave 
function to

$$
\int_{0}^{\infty} d r r^{2}\left|\phi_{d}^{\sigma_{d}}(r)\right|^{2}=1
$$

Defining the breakup component

$$
\psi_{\sigma_{d}}^{\mathrm{bu}}(r, \mathbf{R})=\psi_{\sigma_{d}}(r, \mathbf{R})-\psi_{\sigma_{d}}^{\mathrm{el}}(r, \mathbf{R}),
$$

and substituting the projected expression

$$
\psi_{\sigma_{d}}(r, \mathbf{R})=\psi_{\sigma_{d}}^{\mathrm{el}}(r, \mathbf{R})+\psi_{\sigma_{d}}^{\mathrm{bu}}(r, \mathbf{R})
$$

into Eq. (12) yields an inhomogeneous equation for $\psi_{\sigma_{d}}^{\mathrm{bu}}$,

$$
\begin{aligned}
{\left[E-H_{n p}-T_{R}-\widetilde{U}(r, \mathbf{R})\right] \psi_{\sigma_{d}}^{\mathrm{bu}}(r, \mathbf{R}) } \\
=\left[\widetilde{U}(r, \mathbf{R})+\left(T_{R}-E_{\mathrm{c} . \mathrm{m} .}\right)\right] \psi_{\sigma_{d}}^{\mathrm{el}}(r, \mathbf{R}) .
\end{aligned}
$$

The breakup component of the wave function thus appears as the solution of an equation in which the corresponding elastic component appears in a source term. It is well known that the adiabatic approximation provides a very good description of the elastic channel. CDCC calculations ${ }^{2}$ also produce elastic wave functions in excellent agreement with those of the adiabatic approximation. For the purposes of evaluating the source term in Eq. (23) we can thus take, with some confidence, the elastic part of the exact wave function $\psi_{\sigma_{d}}^{\mathrm{el}}$ to be that of an adiabatic calculation $\psi_{\sigma_{d}}^{A d, \text { el }}$, obtained from the solution of Eq. (7), as in Eqs. (18) and (20), by projection:

$$
\psi_{\sigma_{d}}^{A d, \mathrm{el}}(r, \mathbf{R})=\sum_{\sigma_{d}^{\prime}} \psi_{\sigma_{d}^{\prime} \sigma_{d}}^{A d, \mathrm{el}}(\mathbf{R}) \phi_{d}^{\sigma_{d}^{\prime}}(r)
$$

where

$$
\psi_{\sigma_{d}^{\prime} \sigma_{d}}^{A d, \mathrm{el}}(\mathbf{R})=\int_{0}^{\infty} d r r^{2} \phi_{d}^{\sigma_{d}^{\prime}}(r) \psi_{\sigma_{d}}^{A d}(r, \mathbf{R})
$$

Our goal is to replace the $n-p$ Hamiltonian $H_{n p}$ by a number, or function independent of $r$, as in the adiabatic approximation, so as to remove the dynamical dependence on $r$ and preserve the simplicity of the calculation. For the moment we will denote this average breakup energy by $\bar{\varepsilon}$ and will discuss our prescription for $\bar{\varepsilon}$ in the following.

These steps define what we refer to as the quasiadiabatic approximation to the breakup component of the threebody wave function $\psi_{\sigma_{d}}^{Q a, \text { bu }}$, which satisfies the equation

$$
\begin{aligned}
{\left[E-\bar{\varepsilon}-T_{R}\right.} & -\widetilde{U}(r, \mathbf{R})] \psi_{\sigma_{d}}^{Q a, \mathrm{bu}}(r, \mathbf{R}) \\
= & {\left[\widetilde{U}(r, \mathbf{R})+\left(T_{R}-E_{\mathrm{c} . \mathrm{m} .}\right)\right] \psi_{\sigma_{d}}^{A d, \mathrm{el}}(r, \mathbf{R}) . }
\end{aligned}
$$

Making a partial-wave expansion of the functions involved, namely,

$$
\psi_{\sigma_{d}^{\prime} \sigma_{d}}^{i}(r, \mathbf{R})=\frac{4 \pi}{k R} \sum_{J L M \Lambda}\left(L \Lambda 1 \sigma_{d} \mid J M\right)\left(L \Lambda 1 \sigma_{d}^{\prime} \mid J M\right) i^{L} Y_{L}^{\Lambda}(\hat{k})^{*} Y_{L}^{\Lambda}(\hat{R}) \chi_{J L}^{i}(r, R)
$$

where $i$ represents $(Q a, \mathrm{bu})$ or $(A d, \mathrm{el})$, the quasiadiabatic equation at the partial-wave level is

$$
\left(\frac{d^{2}}{d R^{2}}+k^{2}-\frac{L(L+1)}{R^{2}}-\frac{2 \mu}{\hbar^{2}}\left[\widetilde{U}_{J L}(r, R)+\bar{\varepsilon}\right]\right) \chi_{J_{L}}^{Q a, \mathrm{bu}}(r, R)=\frac{2 \mu}{\hbar^{2}}\left[\widetilde{U}_{J L}(r, R)+\left(T_{R}-E_{\mathrm{c} . \mathrm{m} .}\right)_{L}\right] \chi_{J L}^{A d, \mathrm{el}}(R),
$$

where $k$ is the asymptotic wave number corresponding to $E_{\text {c.m. }}, \mu$ the $n-p$ target reduced mass, $\widetilde{U}_{J L}$ the $J, L$ matrix element of $\widetilde{U}$,

$$
\widetilde{U}_{J L}(r, R)=\widetilde{U}^{c}(r, R)+\widetilde{U}^{\text {so }}(r, R)\langle J L|\mathbf{L} \cdot \mathbf{S}| J L\rangle,
$$

and

$$
\left(T_{R}-E_{\text {c.m. }}\right)_{L} \chi_{J L}^{A d, \mathrm{el}}(r, R)=-\frac{\hbar^{2}}{2 \mu}\left(\frac{d^{2}}{d R^{2}}+k^{2}-\frac{L(L+1)}{R^{2}}\right) \chi_{J L}^{A d, \mathrm{el}}(r, R) .
$$

When calculating the transfer reaction in zero-range approximation, only the coincidence wave functions $\chi_{J_{L}}^{Q a, \text { bu }}(r=0, R)$ are required.

\section{The mean energy prescription}

We see from Eq. (28) that $\bar{\varepsilon}$ should be interpreted as the mean change in the $n$ - $p$ center-of-mass energy due to the important breakup components in the wave function. We can, in general, allow $\bar{\varepsilon}$ to be $J, L$, and $R$ dependent. At a lower energy, Amakawa, Austern, and Vincent used a particularly simple exponential prescription ${ }^{7}$ for $\bar{\varepsilon}$, suggested by the asymptotic $(R \rightarrow \infty)$ mean breakup energy obtained from CDCC calculations near $20 \mathrm{MeV}$. We take the view that we are examining the corrections to the lowest-order Johnson-Soper adiabatic treatment and estimate $\bar{\varepsilon}$ consistently within this model. Consequently, we take the breakup part of the adiabatic wave function $\psi_{\sigma_{d}}^{A d}$, bu as providing our lowest-order approximation to the spectrum of $n-p$ relative motion states excited, as a function of $R$, in a given state $J, L$. We replace $\bar{\varepsilon}$, in each $J, L$ partial wave, by

$$
\bar{\varepsilon}_{J L}(R)=\frac{\left\langle\chi_{J L}^{A d, \mathrm{bu}}(r, R) \phi_{d}(r)\left|H_{n p}\right| \chi_{J L}^{A d, \mathrm{bu}}(r, R) \phi_{d}(r)\right\rangle}{\left\langle\chi_{J L}^{A d, \mathrm{bu}}(r, R) \phi_{d}(r) \mid \chi_{J L}^{A d, \mathrm{bu}}(r, R) \phi_{d}(r)\right\rangle},
$$


where the bra-ket denotes the radial integration over $r$. Equation (31) assigns to $\bar{\varepsilon}$ the real expectation value of $H_{n p}$ in the breakup continuum excited in the adiabatic approximation. This equation can also be written, with $\mu_{n p}$ the reduced mass of the $n-p$ pair, as

$$
\bar{\varepsilon}_{J L}(R)=-\epsilon_{d}+\frac{\hbar^{2}}{2 \mu_{n p}} \frac{\left\langle\phi_{d}(r)(\partial / \partial r) \chi_{J L}^{A d, \mathrm{bu}}(r, R) \mid \phi_{d}(r)(\partial / \partial r) \chi_{J L}^{A d, \mathrm{bu}}(r, R)\right\rangle}{\left\langle\chi_{J L}^{A d, \mathrm{bu}}(r, R) \phi_{d}(r) \mid \chi_{J L}^{A d, \mathrm{bu}}(r, R) \phi_{d}(r)\right\rangle}
$$

in which form the second term, representing the corrections to the adiabatic approximation, is seen to arise directly from a consideration of the $r$ dependence of $\chi_{J L}^{A d, \mathrm{bu}}$.

The mean energy $\bar{\varepsilon}_{J L}(R)$ is a complicated function of $R$ at short distances in every $J$ and $L$ state. At values of $R$ well outside the nucleus, $\bar{\varepsilon}_{J L}(R)$ becomes constant. Its dependence on $J$ and $L$ are shown in Fig. 2. In fact, the transfer calculations shown later in this paper show little sensitivity to whether the full $\bar{\varepsilon}_{J L}(R)$ or its asymptotic $(R \rightarrow \infty)$ value is used.

\section{THE FINAL-STATE PROTON OPTICAL MODEL POTENTIAL}

The final-state proton optical model calculations used a potential of the form of Eq. (14), where

$$
\begin{aligned}
& U_{p}^{c}\left(r_{p}\right)=-V_{\mathrm{CR}} f_{\mathrm{CR}}\left(r_{p}\right)-i V_{\mathrm{CI}} f_{\mathrm{CI}}\left(r_{p}\right), \\
& U_{p}^{\mathrm{sO}}\left(r_{p}\right)=2\left[V_{\mathrm{SOR}} \frac{1}{r_{p}} \frac{d}{d r_{p}} f_{\mathrm{SOR}}\left(r_{p}\right)\right. \\
&\left.+i V_{\mathrm{SOI}} \frac{1}{r_{p}} \frac{d}{d r_{p}} f_{\mathrm{SOI}}\left(r_{p}\right)\right],
\end{aligned}
$$

and $f_{i}$ is the conventional Woods-Saxon form factor

$$
f_{i}\left(r_{p}\right)=\left[1+\exp \left[\frac{r_{p}-r_{0}^{i} A^{1 / 3}}{a_{i}}\right)\right]^{-1} \text {. }
$$

The inclusion of an imaginary spin-orbit term provides a much superior fit to the data near $100 \mathrm{MeV}$. The potential parameters were adjusted to minimize $\chi^{2}$ for the

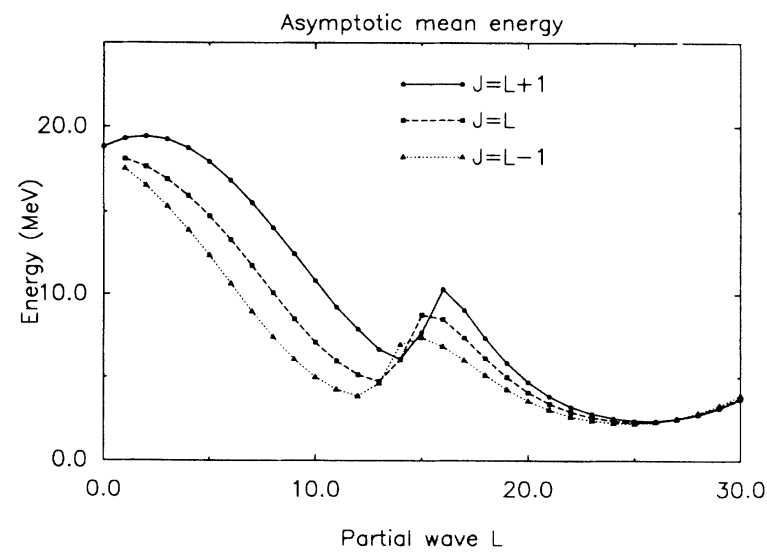

FIG. 2. Large $R$ behavior of the mean energy $\bar{\varepsilon}_{J L}(R)$ of Eq. (31) as a function of the $n-p$ center-of-mass partial wave $L$. comparison of the optical model calculation with the measured cross section and vector analyzing power angular distributions. The calculations were made with the program SNOOPY8Q. ${ }^{16}$ The final values of the parameters are given in Table $I$ and the resulting angular distributions are shown, together with the data, in Fig. 3.

\section{CALCULATIONS WITH THE ADWA MODEL}

The ADWA calculations were finite range, including transfer from both the $S$ and $D$ states of the deuteron, and contained corrections for nonlocality in the deuteron and proton channels. ${ }^{18}$ The nonlocality parameters were taken from Ref. 3. Calculations were performed using the program TWOFNR. ${ }^{19}$ The bound neutron wave function was calculated in a Woods-Saxon well with shape parameters $r_{0}=1.25 \mathrm{fm}$ and $a=0.65 \mathrm{fm}$, and a spin-orbit potential depth $V_{\text {SOR }}=6.0 \mathrm{MeV}$. The real well depth was adjusted to reproduce the neutron separation energy. The adiabatic three-body wave function $\bar{\psi}_{\sigma_{d}}^{A d}$, Eq. (9), was calculated from the underlying nucleon-nucleus optical potentials of Becchetti and Greenlees. ${ }^{20}$ The folding integral, Eq. (11), was carried out for the Reid soft-core interaction and deuteron wave function, ${ }^{21}$ and the resulting adiabatic distorting potentials $U^{A d}$ fitted to conven-

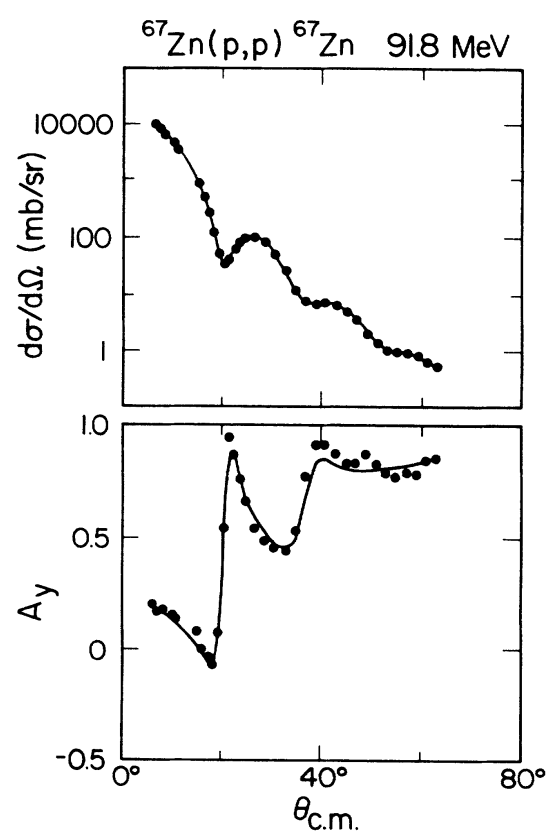

FIG. 3. Angular distributions of the cross section and analyzing power for $91.8-\mathrm{MeV}$ protons scattering from ${ }^{67} \mathrm{Zn}$. The curves are best-fit optical model calculations. 
TABLE I. Proton and adiabatic distorting potential parameters (units are in $\mathrm{MeV}$ and in fm).

\begin{tabular}{lccc}
\hline & & $\begin{array}{c}\text { Optical } \\
\text { potential } \\
p+{ }^{67} \mathrm{Zn}^{\mathrm{a}}\end{array}$ & $\begin{array}{r}\text { Adiabatic } \\
\text { potential } \\
d+{ }^{66} \mathrm{Zn}^{\mathrm{b}}\end{array}$ \\
\hline Energy & & 91.8 & 88.2 \\
Real central & $V$ & 27.78 & 83.857 \\
& $r$ & 1.251 & 1.161 \\
Imaginary central (volume) & $a$ & 0.694 & 0.798 \\
& & & 15.952 \\
Real spin-orbit & $r$ & 7.04 & 1.378 \\
& $a$ & 1.473 & 0.6177 \\
Imaginary spin orbit & & 0.604 & 6.2 \\
& $V$ & 4.941 & 1.01 \\
& $r$ & 1.065 & 0.75 \\
Coulomb radius & $a$ & 0.692 & \\
\hline \hline
\end{tabular}

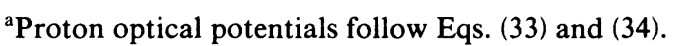

${ }^{b}$ Deuteron adiabatic potential form factor follows Ref. 17.

tional Woods-Saxon potential form factors. These potential parameters are listed in Table I.

Angular distributions of the cross section, deuteron vector $\left(A_{y}\right)$ and tensor $\left(A_{y y}\right)$ analyzing powers and proton polarization $(p)$ are shown in Fig. 4 along with the ADWA calculations. The spectroscopic factor for these calculations was chosen to be 0.20 , which provides a good overall description of the size and exponential decline of the differential cross section. Problems with near-side interference are not so apparent here since the cross section represents a sum of oscillating terms, each of different phase and period. The most significant deviations are forward of $25^{\circ}$.

The lack of a well-developed near-side amplitude is most evident in the spin observables, where the oscillations clearly present in the measurements are essentially absent from the calculations. General trends, such as the sign, magnitude, and average slopes of the angular distributions, depend on the dynamics of the dominant far-side contribution and are qualitatively reproduced.

Figure 5 shows the deviations from the simple relationships of Eqs. (1) and (2). If we define

$$
\tilde{A}_{y}=-\left(2+A_{y y}\right) / 3 \text { and } \widetilde{p}=-\left(1+2 A_{y y}\right) / 3,
$$

then the deviations may be expressed as $A_{y}-\tilde{A}_{y}$ and $p-\tilde{p}$. The $A_{y}-\widetilde{A}_{y}$ calculations for ${ }^{66} \mathrm{Zn}$ differ in two ways from those for ${ }^{116} \mathrm{Sn}$ (see Ref. 5). First, a greater fraction of the difference function is described by the ADWA calculations. The large values backward of $40^{\circ}$ are attributable almost entirely to the deuteron $D$ state. Second, the difference function shows an interference pat- tern (which may be increasing with smaller target mass and $l$ transfer). There is still a factor-of- 2 discrepancy in the middle of the angular range which could be due to either insufficient spin flip in the deuteron channel or the effects of missing strength in the near-side amplitude.

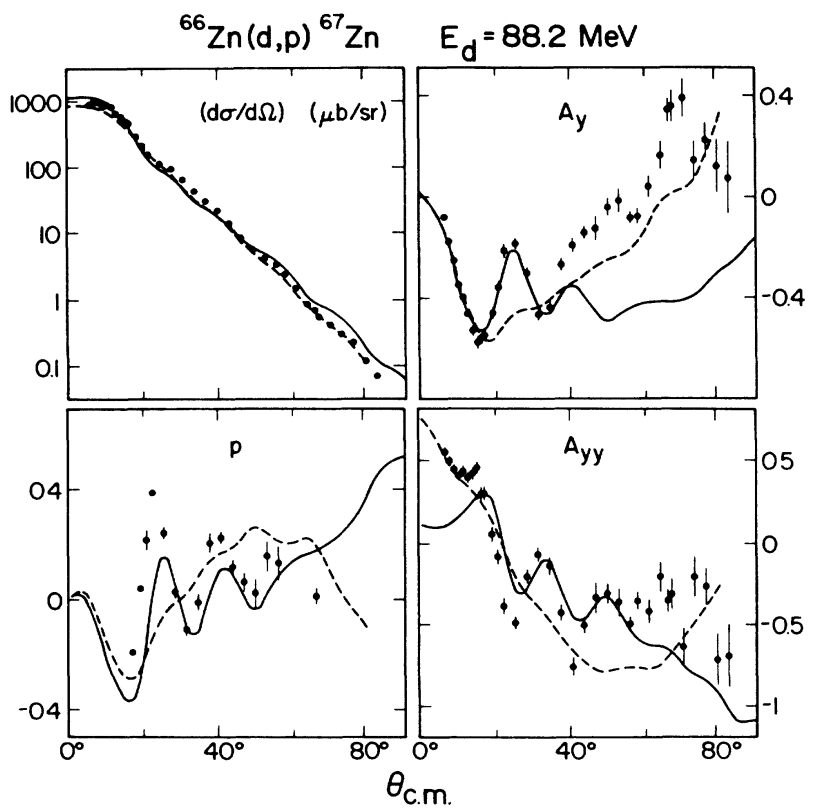

FIG. 4. Angular distributions of the cross section, vector $\left(A_{y}\right)$ and tensor $\left(A_{y y}\right)$ analyzing powers, and the outgoing polarization $(p)$ for the ${ }^{66} \mathrm{Zn}(d, p)^{67} \mathrm{Zn}$ ground-state transition. The dashed curves represent adiabatic calculations; the solid curves represent quasiadiabatic calculations. 


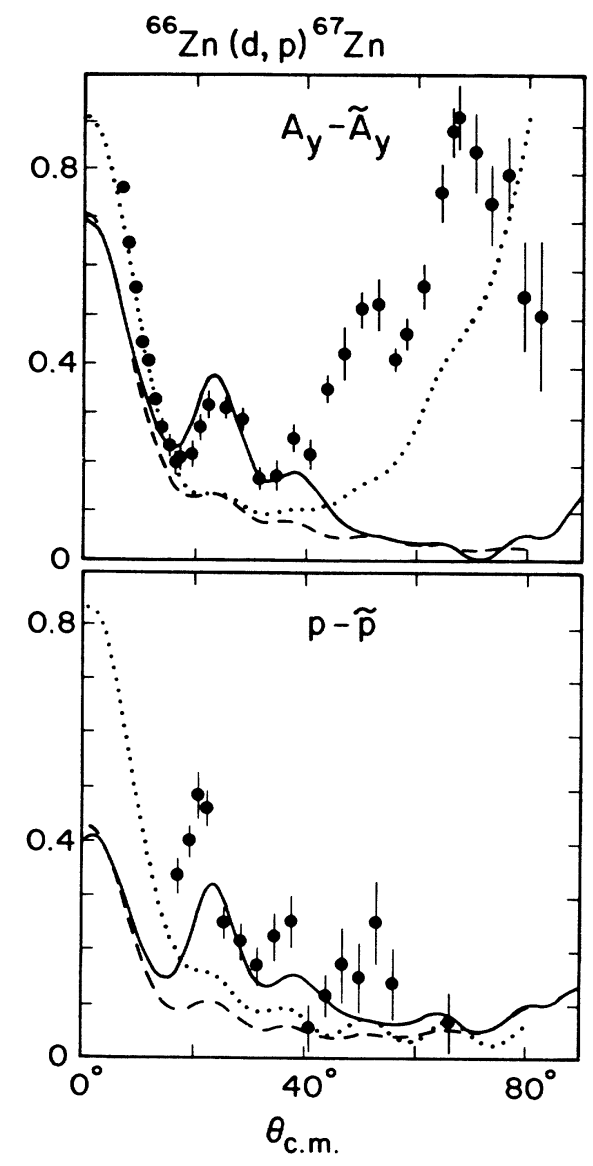

FIG. 5. Angular distributions of the difference functions $A_{y}-\widetilde{A}_{y}$ and $p-\widetilde{p}$ for the ${ }^{66} \mathrm{Zn}(d, p)^{67} \mathrm{Zn}$ ground-state transition. The long-dashed (short-dashed) curve represents adiabatic calculations without (with) the deuteron $D$ state. The solid curve représents quasiadiabatic calculations (without the $D$ state).

Also shown in Fig. 5 are new measurements involving the proton polarization. Here the effects on $p-\widetilde{p}$ of the deuteron $D$ state are significant only at very forward angles where the assumption of far-side dominance that gives rise to Eq. (1) cannot be supported. Again, an interference pattern is present in the difference function, and the calculation falls short of the data by about a factor of 2. Since in this case the difference is not sensitive to deuteron channel spin-flip effects, the combined evidence points toward a missing near-side strength as the major difficulty with these calculations.

\section{EMPIRICAL EXTRACTION OF THE NEAR-SIDE AMPLITUDE}

Following the discussion in Johnson, Stephenson, and Tostevin, ${ }^{6}$ we can associate the two partial cross sections, $(d \sigma / d \Omega)_{0}$ and $(d \sigma / d \Omega)_{-1}$, with the dominant far-side projection of amplitudes with quantum numbers $\left\langle m, \sigma_{p} \mid \sigma_{d}\right\rangle=\left\langle\frac{5}{2}, \frac{1}{2} \mid 0\right\rangle$ and $\left\langle\frac{5}{2},-\frac{1}{2} \mid-1\right\rangle$, respectively. In the ${ }^{116} \mathrm{Sn}$ study, these two amplitudes were successfully modeled by an expression based on simple poles in the upper and lower halves of the $l$ plane. This generated nearly exponential angular distributions for the far-side and near-side amplitudes, except for a rolloff at small angles governed by the order of the singularity.

The measurements of $(d \sigma / d \Omega)_{0}$ and $(d \sigma / d \Omega)_{-1}$ for ${ }^{66} \mathrm{Zn}(d, p)^{67} \mathrm{Zn}$ are shown in Fig. 6. For $(d \sigma / d \Omega)_{0}$ the superimposed interference pattern is strongest at the middle of the angular range and becomes weaker at the ends, while for $(d \sigma / d \Omega)_{-1}$ the opposite is true. Thus it is already clear that an exponentially falling near-side amplitude will be unable to satisfactorily model these measurements. We must abandon our simple pole model if we are to make a useful quantitative estimate of the required near-side strength. As a result, we will not be able to estimate the partial wave which makes the largest contribution to the required near-side amplitude, as was done in Ref. 6.

To obtain an empirical representation of the polarized cross sections, we extended the fitting function of Ref. 6 to be

$$
\begin{aligned}
\varphi_{\mathrm{far}}= & \frac{\theta^{\mu-1}}{\sqrt{\sin \theta}} \exp \left(-\Gamma \theta+\Upsilon \theta^{2}\right), \\
\varphi_{\text {near }}= & \frac{\theta^{v-1}}{\sqrt{\sin \theta}}\left[\exp \left(-\Gamma^{\prime} \theta+\Upsilon^{\prime} \theta^{2}\right)+R \exp \left(-\Gamma^{\prime \prime} \theta\right)\right], \\
\frac{d \sigma}{d \Omega}= & A^{2} \varphi_{\mathrm{far}}^{2}+\left(a^{2}+\bar{a}^{2}\right) \varphi_{\text {near }}^{2} \\
& +2 A[a \cos (\Lambda \theta)+\bar{a} \sin (\Lambda \theta)] \varphi_{\mathrm{far}} \varphi_{\text {near }} .
\end{aligned}
$$

The parameters required to model $(d \sigma / d \Omega)_{0}$ and $(d \sigma / d \Omega)_{-1}$ are listed in Table II, and the model curves

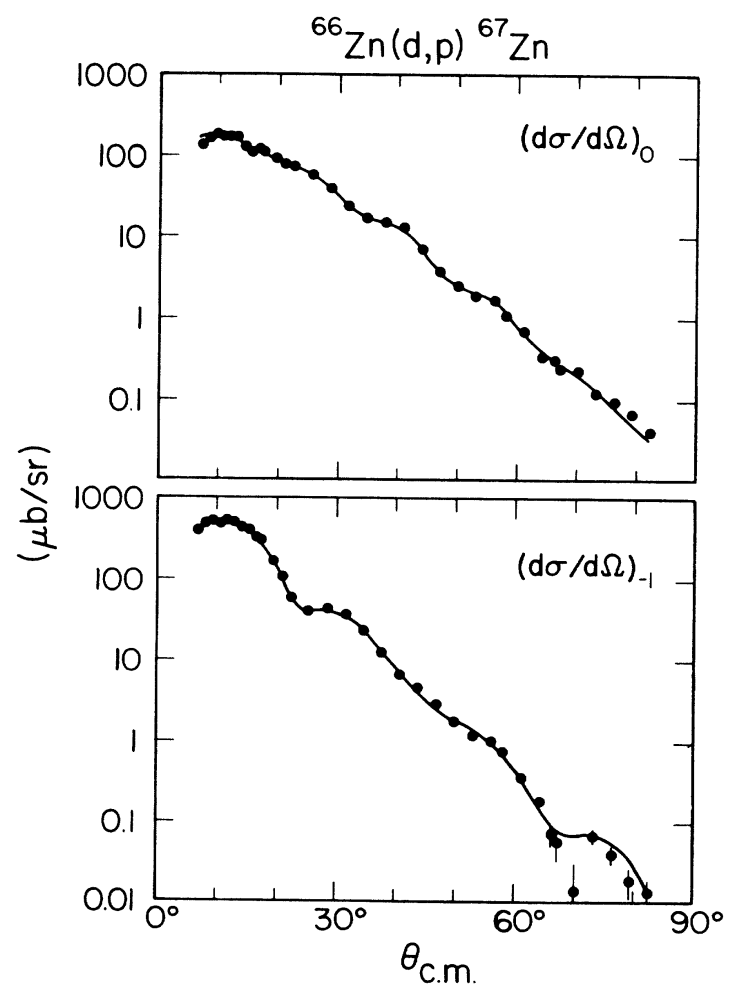

FIG. 6. Angular distributions of the partial cross sections $(d \sigma / d \Omega)_{0}$ and $(d \sigma / d \Omega)_{-1}$ in units of $\mu \mathrm{b} / \mathrm{sr}$. The curves were generated by Eq. (36) using the parameters of Table II. 
TABLE II. Model amplitude parameters.

\begin{tabular}{lcc}
\hline & $(d \sigma / d \Omega)_{0}$ & $(d \sigma / d \Omega)_{-1}$ \\
\hline$A$ & $57.7 \pm 1.7$ & $119.0 \pm 2.2$ \\
$a$ & $-0.09 \pm 0.18$ & $-2.5 \pm 1.4$ \\
$\bar{a}$ & $-0.06 \pm 0.14$ & $-4.6 \pm 2.7$ \\
$\Gamma$ & $3.62 \pm 0.10$ & $5.120 \pm 0.042$ \\
$\Upsilon$ & $-0.463 \pm 0.075$ & \\
$\Gamma^{\prime}$ & $-6.5 \pm 5.0$ & $-6.1 \pm 3.3$ \\
$\Upsilon^{\prime}$ & $-6.7 \pm 3.2$ & $-16.9 \pm 5.1$ \\
$\Lambda$ & $22.87 \pm 0.82$ & $17.20 \pm 0.30$ \\
$R$ & & $0.19 \pm 0.34$ \\
$\Gamma^{\prime \prime}$ & & $2.28 \pm 0.25$ \\
$\chi^{2} / v$ & 3.2 & 5.9 \\
\hline
\end{tabular}

are shown in Fig. 6. Each far- and near-side amplitude contains an additional $\theta^{2}$ dependence. For $(d \sigma / d \Omega)_{0}$ this additional term is needed in the far-side amplitude to reproduce a gentle change in the slope of the cross section with angle. When this $\theta^{2}$ term becomes large, as it does in the corresponding near-side amplitude, that amplitude takes a Gaussian shape, and the slope parameter $\Gamma^{\prime}$ becomes negative and controls the angle at which the Gaussian is at a maximum. For $(d \sigma / d \Omega)_{-1}$ an exponential far-side amplitude is adequate, and the small-angle interference pattern is well reproduced by a Gaussian near-side term. To restore the interference pattern at large scattering angles, a second exponential piece with a small slope is added to the near-side amplitude.

Figure 7 shows two bands for $(d \sigma / d \Omega)_{0}$ and $(d \sigma / d \Omega)_{-1}$ corresponding to the limits of one standard deviation in the model of the far- and near-side amplitudes. The size of these bands has been increased by $\sqrt{\chi^{2} / v}$ to compensate for imperfections in the model. The full error matrix was used; correlations among parameters often produce a small error band even when the near-side coefficients ( $a$ and $\bar{a}$ ) have individual errors that include zero. The Gaussian shapes of the near-side amplitude are clearly evident in the shape of the error band. (The use of purely exponential amplitudes results in a significantly poorer reproduction of the polarized cross sections, and error bands consistent with those shown in Fig. 7.)

\section{CALCULATIONS WITH THE QUASIADIABATIC MODEL}

The quasiadiabatic transfer reaction calculations were performed in zero-range approximation, but included corrections for nonlocality in the deuteron and proton channels. The nonlocality parameters and neutron bound-state wave function were the same as for the ADWA calculations. Calculations were performed using a version of the program TWOFNR, modified to read externally calculated wave functions. The inhomogeneous quasiadiabatic radial equation, Eq. (28), was solved, at coincidence, with $\widetilde{U}(r=0, R)$ derived from the nucleon-nucleus optical potentials of Becchetti and Greenlees. The mean energy prescription of Eqs. (31) and (32) was used, evaluated numerically from the solu- tion of the adiabatic equation.

The calculated quasiadiabatic angular distributions of the cross section, vector $\left(A_{y}\right)$ and tensor $\left(A_{y y}\right)$ analyzing powers, and proton polarization $(p)$ are shown in Fig. 4 (solid curves) together with those of the ADWA calculations (dashed curves). The spectroscopic factor for these calculations was taken to be 0.30 . While the cross section angular distribution agrees less well with the general slope of the measurements, there is a dramatic improvement in the ability of the new calculation to match the interference pattern present in the middle of the angular

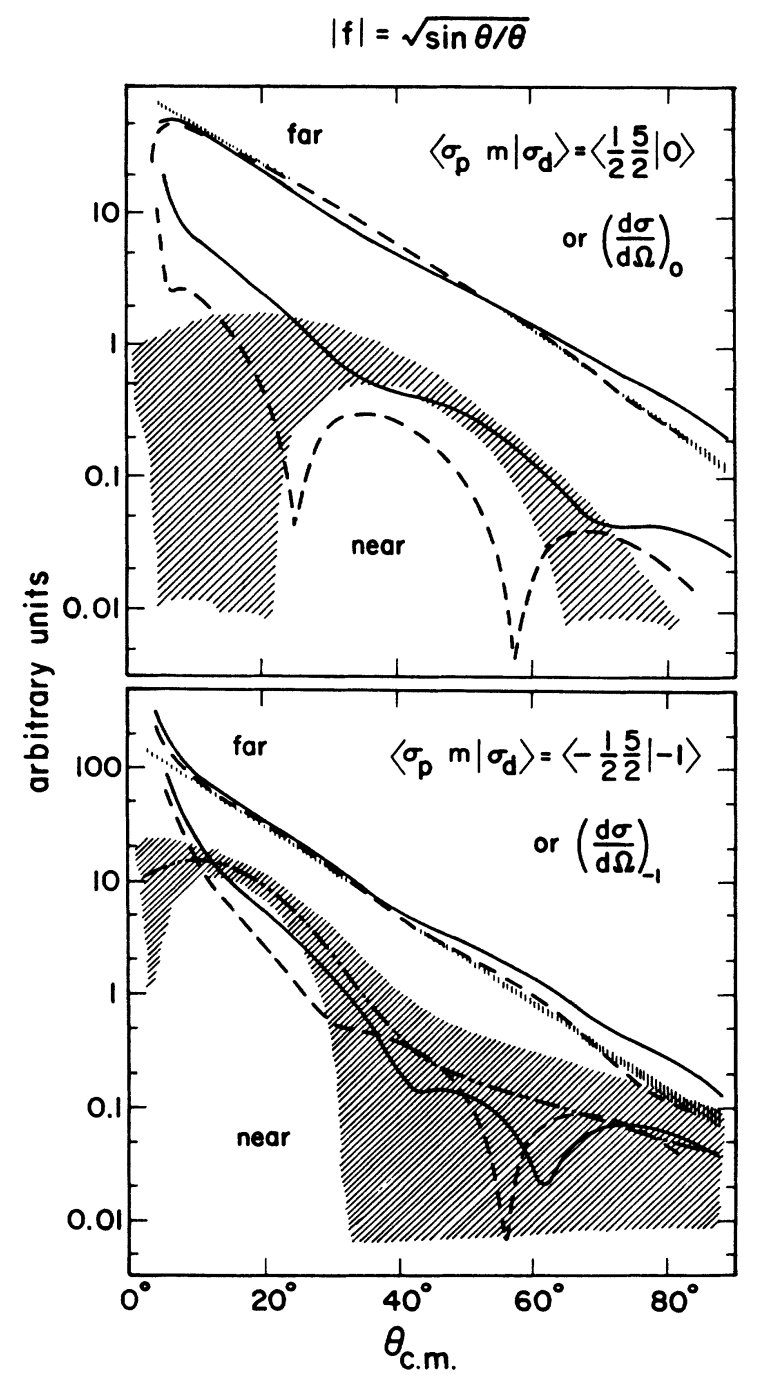

FIG. 7. Angular distributions of the amplitudes $\left\langle m \sigma_{p} \mid \sigma_{d}\right\rangle$ separated into far-side and near-side components. The solid (dashed) curves show this separation for the quasiadiabatic (adiabatic) calculation. These amplitudes have been multiplied by $\sqrt{\sin \theta} / \theta$ to emphasize the linearity of the far-side component. The far-side and near-side amplitudes extracted from the partial cross-section angular distributions of Fig. 6 are indicated by hatched regions that span one standard deviation above and below each amplitude. The far-side uses vertical hatching and the near-side diagonal hatching. For $(d \sigma / d \Omega)_{-1}$ a dashdouble-dotted line has been added for clarity to represent the experimental near-side amplitude. 
range for the spin observables. The greatest improvement comes for the polarization; the best reproduction of the size of the interference pattern is for the vector analyzing power. In comparing these calculations to the full range of the measurements, effects of the deuteron $D$ state, which are known to be present at forward angles in the tensor analyzing power, are missing. So a failure at this point to reproduce the general trend of the spin observables in this angle range should not be regarded as a problem with the quasiadiabatic prescription.

Figure 5 shows the calculated deviations from the simple relationships of Eqs. (1) and (2). Here the most striking improvements are the significantly larger values in the middle angle range, and the ability to reproduce much of the interference pattern in that angular range. Again, the $D$-state effects, responsible in the adiabatic calculations for the small- and large-angle rise, are absent, and the new calculation follows the trend of the $S$ wave part of the adiabatic calculation at small and large angles. It remains to be seen whether the addition of the deuteron $D$-state component can reproduce the observed interference oscillations at the larger angles.

Since the deuteron $D$ state is omitted from the quasiadiabatic calculation, agreement in detail with the angular distributions is not possible. Perhaps a better indication of the ability of the quasiadiabatic scheme to model the reaction mechanism correctly lies in the comparison with the size of the far-side and near-side amplitudes described in the preceding section. The far- and near-side components of a given element of the scattering matrix may be calculated by changing the partial-wave expansion according to Eq. (2) of Ref. 6. Far each of the partial cross sections, $(d \sigma / d \Omega)_{0}$ and $(d \sigma / d \Omega)_{-1}$, the largest contribution to the scattering matrix element was chosen for this separation. This was $\left\langle m \sigma_{p} \mid \sigma_{d}\right\rangle$ $=\left\langle\frac{5}{2}, \frac{1}{2} \mid 0\right\rangle$ and $\left\langle\frac{5}{2},-\frac{1}{2} \mid-1\right\rangle$ for the two partial cross sections, respectively. The dashed lines in Fig. 7 show the far- and near-side amplitudes for the adiabatic calculation, renormalized to best reproduce the far-side term. The solid lines show a similar result for the quasiadiabatic case. For both partial cross sections, the near-side term is larger in the quasiadiabatic calculation. For $(d \sigma / d \Omega)_{0}$, the larger near-side term agrees well with the empirical amplitude. Near $0^{\circ}$, the irregular Legendre function used in the separation makes both far- and near-side calculations tend to infinity, and there we expect disagreement with the empirical formula. For $(d \sigma / d \Omega)_{-1}$, the agreement is less quantitative, in part because of the tendency of the small-angle calculations to rise, and because of the poor knowledge of the large-angle near-side amplitudes (despite a clear interference pattern in the large-angle partial cross section). In general, the scattering energies associated with the quasiadiabatic calculation result in larger near-side amplitudes, in agreement with what is required experimentally.

\section{CONCLUSIONS}

Large $l$ transfer $j=l-\frac{1}{2}(d, p)$ transitions at energies near $100 \mathrm{MeV}$ provide a particularly stringent test of three-body reaction models. The spin coupling involved and small spin-flip terms present in this energy regime ensure that certain amplitudes that might be expected to contribute in the process are not significant. The result is that the surviving amplitudes, and their interference with other smaller components, can be probed at a level hitherto not thought possible.

In this paper we report new measurements of the spin observables for the $j=l-\frac{1}{2}$ transition to the $\frac{5}{2}^{-}$ground state in the ${ }^{66} \mathrm{Zn}(d, p){ }^{67} \mathrm{Zn}$ reaction. These data were taken for the purpose of exploring further the inadequacies in the three-body treatment of transfer reactions provided by the adiabatic distorted-wave approximation model. Problems noted previously for the ${ }^{116} \mathrm{Sn}(d, p){ }^{117} \mathrm{Sn}$ reaction were also evident in the present data. These included disagreements with theoretical relationships, exact in the limit of far-side dominance, that ADWA calculations indicate should be nearly satisfied, and the presence of a near-side/far-side interference pattern absent in the theoretical calculations. The nearly overlapping features of the two available sets of data indicate that these difficulties are likely to be common characteristics of $(d, p)$ reactions at these bombarding energies, and to depend little on target nucleus or $l$ transfer, provided the $l$ transfer satisfies closely the requirements of angular momentum matching.

Unlike the ${ }^{116} \mathrm{Sn}$ study, the ${ }^{66} \mathrm{Zn}$ measurements showed interference patterns in the difference functions, such as $A_{y}-\tilde{A}_{y}$. Interference patterns in the polarized cross sections, $(d \sigma / d \Omega)_{0}$ and $(d \sigma / d \Omega)_{-1}$, had more complicated angular dependences. It was no longer possible to model the missing near-side amplitudes using a formula of the single-pole form. Nevertheless, a phenomenological model could be used from which the size, or at least an upper limit, for the missing amplitude could be obtained as a function of scattering angle.

The earlier exhaustive analysis of the amplitude structure for ${ }^{116} \mathrm{Sn}$ suggested that the inadequacy with the ADWA might lie with the fixed value of the $n-p$ centerof-mass energy assumed in the adiabatic model. Using the quasiadiabatic approximation, extended to include spin-orbit interactions and a plausible (partial-wave and radially dependent) mean breakup energy prescription, we have taken these modified center-of-mass energy systematics into account. This leads to a calculational scheme in which the breakup portion of the adiabatic wave function is replaced by a breakup component calculated using a more realistic center-of-mass energy. Zerorange calculations made with this new scheme indicate that the two major aspects of the discrepancy noted previously, namely the failure to obey the far-side dominant relationships [Eqs. (1) and (2)] and the presence of a large near-side amplitude, are essentially explained. Clearly the calculations need to be extended to include finiterange and $D$-state effects, and should cover a number of $(d, p)$ transitions, in order to assess the nature of any systematic improvements over the simpler ADWA model. The work reported here suggests, however, that a more complete calculation with the three-body framework, such as the CDCC method, may be able to reproduce in detail the reaction data. 


\section{ACKNOWLEDGMENTS}

We acknowledge many useful discussions with Dr. $\mathbf{M}$. Yahiro, in particular on the subject of mean breakup energy prescriptions, during the course of this work. The financial support of the Science and Engineering Research Council (U.K.) in the form of Grants GR/F/4105.1 and GR/F/1086.6 (for J.A.T. and R.C.J.), the University of Surrey (D.A.C.), and the National Science Foundation is gratefully acknowledged.
*Present address: Energy Studies Unit, Department of Physics, University of Exeter, Devon, United Kingdom.

${ }^{1}$ N. Austern, Y. Iseri, M. Kamimura, M. Kawai, G. Rawitscher, and M. Yahiro, Phys. Rep. 154, 125 (1987).

${ }^{2}$ M. Kamimura, M. Yahiro, Y. Iseri, H. Kameyama, Y. Sakuragi, and M. Kawai, Prog. Theor. Phys. Suppl. 89, 1 (1986).

${ }^{3}$ V. R. Cupps, J. D. Brown, C. C. Foster, W. P. Jones, D. W. Miller, H. Nann, P. Schwandt, E. J. Stephenson, and J. A. Tostevin, Nucl. Phys. A469, 445 (1987).

${ }^{4}$ E. J. Stephenson, V. R. Cupps, J. A. Tostevin, R. C. Johnson, J. D. Brown, C. C. Foster, W. P. Jones, D. W. Miller, H. Nann, and P. Schwandt, Nucl. Phys. A469, 467 (1987).

${ }^{5}$ E. J. Stephenson, R. C. Johnson, J. A. Tostevin, V. R. Cupps, J. D. Brown, C. C. Foster, J. A. Gering, W. P. Jones, D. A. Low, D. W. Miller, H. Nann, C. Olmer, P. Schwandt, J. W. Seubert, and S. W. Wissink, Phys. Lett. B 171, 358 (1986).

${ }^{6}$ R. C. Johnson, E. J. Stephenson, and J. A. Tostevin, Nucl. Phys. A505, 26 (1989).

${ }^{7}$ H. Amakawa, N. Austern, and C. M. Vincent, Phys. Rev. C 29, 699 (1984).

${ }^{8}$ For a review of the properties of atomic beam polarized ion sources, see W. Haeberli, Annu. Rev. Nucl. Sci. 17, 373 (1967).

${ }^{9}$ P. Schwandt, T. B. Clegg, and W. Haeberli, Nucl. Phys. A163, 432 (1971); based on data available in D. C. Dodder, G. M.
Hale, N. Jarmie, and K. Witte, Los Alamos Internal Report LA-6389-MS, 1976.

${ }^{10}$ E. J. Stephenson, J. C. Collins, C. C. Foster, D. L. Friesel, W. W. Jacobs, W. P. Jones, M. D. Kaitchuck, P. Schwandt, and W. W. Daehnick, Phys. Rev. C 28, 134 (1983).

${ }^{11}$ B. L. Cohen, Rev. Sci. Instrum. 33, 85 (1962).

${ }^{12}$ W. Bertozzi, M. V. Hynes, C. P. Sargent, C. Creswell, P. C. Dunn, A. Hirsch, M. Leitch, B. Norum, F. N. Rad, and T. Sasanuma, Nucl. Instrum. Methods 141, 457 (1977).

${ }^{13}$ M. Yahiro, Y. Iseri, M. Kamimura, M. Nakano, and M. Tanifuji, in Proceedings of the International Workshop on Deuteron Involving Reactions and Polarization Phenomena, Tsukuba, 1985, edited by Y. Aoki and K. Yagi (World Scientific, Singapore, 1986), p. 45.

${ }^{14}$ R. C. Johnson and P. J. R. Soper, Phys. Rev. C 1, 56 (1970).

${ }^{15}$ R. C. Johnson and P. C. Tandy, Nucl. Phys. A235, 56 (1974).

${ }^{16}$ P. Schwandt, Computer Program SNOOPY8Q, Indiana University Cyclotron Facility Internal Report 85-9, 1985.

${ }^{17}$ P. Schwandt and W. Haeberli, Nucl. Phys. A123, 401 (1969).

${ }^{18}$ F. Perey and B. Buck, Nucl. Phys. 32, 353 (1962).

${ }^{19}$ M. Igarashi, M. Toyama, and N. Kishida, Computer program TWOFNR, private communication.

${ }^{20}$ F. D. Becchetti, Jr. and G. W. Greenlees, Phys. Rev. 182, 1190 (1969).

${ }^{21}$ R. V. Reid, Jr., Ann. Phys. (N.Y.) 50, 411 (1968). 